\title{
Bug Search Subtest (WPPSI-IV)
}

National Cancer Institute

\section{Source}

National Cancer Institute. Bug Search Subtest (WPPSI-IV). NCI Thesaurus. Code

C120354.

A subtest of the Wechsler Preschool and Primary Scale of Intelligence, 4th Edition that measures short-term visual memory, visual-motor coordination, cognitive flexibility, visual discrimination, and concentration. Working within a specified time limit, the child marks the bug in the search group that matches the target bug. 